\title{
Erratum to: Complete genome sequence of a filamentous bacteriophage, RS611, that infects the phytopathogen Ralstonia solanacearum
}

Truong Thi Bich Van $\cdot$ Shohei Yoshida

Kaito Miki • Akihiro Kondo • Kaeko Kamei

Published online: 28 February 2015

(c) Springer-Verlag Wien 2015

\section{Erratum to: Arch Virol}

DOI 10.1007/s00705-014-2316-8

The authors would like to publish an erratum to correct the typographical error in the figure legend and text.

The sentence from Fig. 1 legend: "....and that of $\phi R S S 1$ was constructed with ORF Finder software." should read as: "and that of $\boldsymbol{\phi R S 6 1 1}$ was constructed with ORF Finder software."

Further, the sentence from second line of right column of the second page: "ORF 4 (66 aa) of $\phi R S 611$ lacked 23 amino acids in the N-terminal region compared with ORF 2 (89 aa) of $\phi R S S 0$ and $\phi R S S 1$ ". Should be: "ORF 4 (66 aa) of $\phi$ RS611 lacked 23 amino acids in the N-terminal region compared with ORF 4 (89 aa) of $\phi R S S 0$ and $\phi R S S 1 . "$

The online version of the original article can be found under doi:10.1007/s00705-014-2316-8.

T. T. B. Van · S. Yoshida $\cdot$ K. Miki $\cdot$ K. Kamei $(\bowtie)$ Department of Biomolecular Engineering, Kyoto Institute of Technology, Matsugasaki, Sakyo-ku, Kyoto 606-8585, Japan e-mail: kame@kit.ac.jp

A. Kondo

Hiyoshi Corporation, 908 Kitanosho, Omihachiman,

Shiga 523-8555, Japan 